\title{
Unitarity of theories containing fractional powers of the d'Alembertian operator
}

\author{
E. C. Marino ${ }^{1}$, Leandro O. Nascimento ${ }^{1,3}$, Van Sérgio Alves ${ }^{1,2}$, and C. Morais Smith $^{3}$ \\ ${ }^{1}$ Instituto de Física, Universidade Federal do Rio de Janeiro, \\ C.P.68528, Rio de Janeiro RJ, 21941-972, Brazil \\ ${ }^{2}$ Faculdade de Física, Universidade Federal do Pará, \\ Av. Augusto Correa 01, 66075-110, Belém, Pará, Brazil \\ ${ }^{3}$ Institute for Theoretical Physics, Utrecht University, \\ Leuvenlaan 4, 3584CE Utrecht, The Netherlands
}

(Dated: July 18, 2018)

\begin{abstract}
We examine the unitarity of a class of generalized Maxwell $\mathrm{U}(1)$ gauge theories in $(2+1) \mathrm{D}$ containing the pseudodifferential operator $\square^{1-\alpha}$, for $\alpha \in[0,1)$. We show that only Quantum Electrodynamics $\left(\mathrm{QED}_{3}\right)$ and its generalization known as Pseudo Quantum Electrodynamics (PQED), for which $\alpha=0$ and $\alpha=1 / 2$, respectively, satisfy unitarity. The latter plays an important role in the description of the electromagnetic interactions of charged particles confined to a plane, such as in graphene or in hetero-junctions displaying the quantum Hall effect.

PACS numbers: 11.15.-q, 11.10.Lm, 11.55.Bq
\end{abstract}

\section{INTRODUCTION}

Unitarity is an important necessary condition for the consistency of any quantum theory. Consider the time evolution operator $U(t, 0)$, defined as

$$
|\Psi(t)\rangle=U(t, 0)|\Psi(0)\rangle,
$$

where $|\Psi(t)\rangle$ is the state-vector at instant $t$. The unitarity of the time-evolution operator, namely the property $U^{\dagger} U=U U^{\dagger}=I$, where $I$ is the identity operator, guarantees that the norm of the state-vectors, chosen equal to one, is preserved in time. Since the state-vector can be expanded in the eigenstates of any observable $A$, it follows that its norm is equal to the sum of the probabilities for the possible outcomes of any measurement of $A$. Unitarity implies that this sum of probabilities remains equal to one at any time, an essential condition for the probabilistic description of a system. For a timeindependent Hamiltonian, we have $U(t, 0)=\exp (-i H t)$. Unitarity then implies that the Hamiltonian is a hermitian operator and therefore the energy eigenvalues are real. This property and the conservation of the sum of probabilities are crucial conditions for the stabillity of a quantum-mechanical system [1].

Another consequence of the unitarity of the timeevolution operator is that the scattering matrix, which connects the asymptotic states after a scattering event to the ones before it, must also be unitary. Assuming the completeness of the asymptotic states, then it follows that the $S$-matrix elements form a matrix representation of a unitary scattering operator $S=1+i T$. Unitarity of the $S$-operator, namely, $S^{\dagger} S=1$, implies

$$
i\left(T^{\dagger}-T\right)=T^{\dagger} T \text {. }
$$

This relation leads to the optical theorem, which relates the forward scattering amplitude to the total cross section of the scatterer. A very convenient way of testing the consistency of a theory is then provided by the opti- cal theorem, which is satisfied by unitary theories (for a nice review about the optical theorem see the Ref. [2]).

In this paper, we examine the unitarity of a class of generalized Maxwell U(1) gauge theories in $(2+1) \mathrm{D}$ by using the optical theorem. For an appropriate choice of the gauge, the equations of motion for these theories are $\square^{1-\alpha} A_{\mu}=0$, for any $\alpha \in[0,1)$. We show that only the choices $\alpha=0$ or $\alpha=1 / 2$ corresponding, respectively, to $\mathrm{QED}_{3}$ and the so-called pseudoQED (PQED) provide a self-consistent solution to the optical theorem. Particularly, the choice $\alpha=1 / 2$ is also consistent with the Huygens principle Ref. [3]. The unitarity of PQED is first proven at the tree level, and then for the interacting case.

The outline of this paper is the following: In Sec. II we revise the PQED and propose its generalization to any $\alpha$. In Sec. III we show that only $\alpha=0$ or $\alpha=1 / 2$ are possible choices in order to obtain a self-consistent solution of the optical theorem. Both cases are considered at the tree level, with no source term in the equation of motion. In Sec. IV we use the RPA approach to show that the version of PQED used to describe the electronic interaction in graphene is also unitary. In Sec. V we adopt perturbation theory up two loop to show that the PQED is an unitary theory.

\section{THE PQED AND ITS GENERALIZATIONS}

\section{A. The Derivation of PQED}

The discovery of condensed matter systems with physical properties that are essentially two-dimensional has fostered the investigation of $(2+1) \mathrm{D}$ theories, which could appropriately describe them. Among these we find the GaAs quantum wells exhibiting the quantum Hall effect, the high-Tc cuprates and graphene [4]. In such systems, a crucial issue is the description of the electronic interaction, which naturally is electromagnetic (EM). For this 
matter, one must consider that the interaction among the electrons is usually mediated by a (spacially) threedimensional field in spite of the fact that the electron kinematics is confined to a plane. For the sake of convenience, simplicity and elegance, however, it is preferable to provide a completely $(2+1)$-dimensional description of the real electromagnetic interaction among the electrons. This is achieved [5] 8] by a theory, coined Pseudo Quantum Electrodynamics (PQED), which was also used in the bosonization of the massless Dirac field in $(2+1) \mathrm{D}$ [9]. Dynamical mass generation for massless electrons also was studied for this model [10].

In this section, for the sake of completeness, we review the main steps of the derivation contained in Ref. [5]. We start from standard $\mathrm{QED}_{4}$, in $(3+1) \mathrm{D}$ :

$$
\mathcal{L}_{Q E D}=-\frac{1}{4} F_{\mu \nu} F^{\mu \nu}-e j_{3+1}^{\mu} A_{\mu}+\mathcal{L}_{m}
$$

where $j_{3+1}^{\mu}$ and $\mathcal{L}_{m}$ are, respectively, the electronic current and kinetic Lagrangian. $A_{\mu}$ is the gauge field, $F^{\mu \nu}$ is the usual field-strength tensor.

The electromagnetic field induces an effective currentcurrent interaction on the electrons, which is captured by the functional (in Euclidean space)

$$
Z_{Q E D}\left[j_{3+1}^{\mu}\right]=Z_{0}^{-1} \int D A_{\mu} \exp \left\{-\int d^{4} \xi \mathcal{L}_{Q E D}\right\},
$$

where $\xi=(x, y, z, \tau)$ and $Z_{0}$ is a normalization constant which guarantees that $Z[0]=1$. The functional integration above can be carried out by including a gauge fixing term, yielding

$$
\begin{aligned}
& Z_{Q E D}\left[j_{3+1}^{\mu}\right]=\exp \left\{-\frac{e^{2}}{2} \int d^{4} \xi d^{4} \xi^{\prime} j_{3+1}^{\mu}(\xi)\right. \\
&\left.\times G_{Q E D}^{\mu \nu}\left(\xi-\xi^{\prime}\right) j_{3+1}^{\nu}\left(\xi^{\prime}\right)\right\},
\end{aligned}
$$

where $G_{Q E D}^{\mu \nu}$ is the Euclidean propagator of the electromagnetic field, which is given by

$$
G_{Q E D}^{\mu \nu}\left(\xi-\xi^{\prime}\right)=\delta^{\mu \nu} \int \frac{d^{4} k}{(2 \pi)^{4}} \frac{e^{i k \cdot\left(\xi-\xi^{\prime}\right)}}{k^{2}}+\mathrm{gt},
$$

where gt stands for "gauge dependent terms". These, by the way, do not contribute for Eq. (5).

We now introduce the fact that the electrons are supposed to move on a plane at $z=0$, thus forming a spatially two-dimensional system. The electronic current, accordingly, is given by

$$
j_{3+1}^{\mu}(\xi)= \begin{cases}j^{\mu}(x, y, \tau) \delta(z), & \mu=0,1,2, \\ 0, & \mu=3 .\end{cases}
$$

Inserting Eq. (7) in Eq. (5) and integrating over $z$ and $z^{\prime}$, we get

$$
\begin{aligned}
& Z_{Q E D}\left[j^{\mu}\right]=\exp \left\{-\int d^{3} \eta d^{3} \eta^{\prime} j^{\mu}(\eta)\right. \\
& \left.\times G_{Q E D}^{\mu \nu}\left(\eta-\eta^{\prime} ; z=z^{\prime}=0\right) j^{\nu}\left(\eta^{\prime}\right)\right\}
\end{aligned}
$$

where $\eta=(x, y, \tau)$ and

$$
G_{Q E D}^{\mu \nu}\left(\eta-\eta^{\prime} ; z=z^{\prime}=0\right)=\frac{\delta^{\mu \nu}}{8 \pi^{2}\left|\eta-\eta^{\prime}\right|^{2}}+\mathrm{gt} .
$$

The expression above is the 4-dimensional QED Euclidean propagator, calculated at $z=z^{\prime}=0$.

Now comes a key step in our derivation. This is the realization that Eq. (9) can be written as a 3-dimensional Fourier integral, namely

$$
\frac{1}{8 \pi^{2}\left|\eta-\eta^{\prime}\right|^{2}}=\int \frac{d^{3} k_{3 D}}{(2 \pi)^{3}} \frac{e^{i k_{3 D} \cdot\left(\eta-\eta^{\prime}\right)}}{4 \sqrt{k_{3 D}^{2}}}
$$

and this is the euclidean propagator of PQED [5], corresponding to the strictly $(2+1)$-dimensional Lagrangian

$$
\mathcal{L}_{P Q E D}=-\frac{1}{4} F_{\mu \nu}\left[\frac{4}{(-\square)^{1 / 2}}\right] F^{\mu \nu}-e j^{\mu} A_{\mu}+\mathcal{L}_{m}
$$

Inserting Eq. (9) and Eq. (10) in Eq. (8), we can immediately realize that

$$
Z_{Q E D}\left[j^{\mu}\right]=Z_{0}^{-1} \int D A_{\mu} \exp \left\{-\int d^{3} \eta \mathcal{L}_{P Q E D}\right\} .
$$

The above derivation shows that all the electronic properties determined by $\mathrm{QED}_{4}$, when projected on a plane are described by a strictly $(2+1)$-dimensional theory, namely PQED. In connection to this point, one could argue whether PQED provides a description of the correlation functions of $\mathrm{QED}_{4}$. The $A_{\mu}$ correlators are generated by coupling an external source $J_{3+1}^{\mu}$ in Eq. (4), namely

$$
j_{3+1}^{\mu} \rightarrow j_{3+1}^{\mu}+J_{3+1}^{\mu}
$$

and subsequently taking functional derivatives of $Z_{Q E D}$ with respect to this source. Assuming it has the same structure as the electronic current given by Eq. (77), it follows that functional derivatives with respect to the $(2+1)$-dimensional external source taken in PQED will generate the projected correlators, as it occurred with the two-point function in Eq. (9).

\section{B. Generalized PQED}

We will consider here a class of theories in $(2+1) \mathrm{D}$, which contain PQED and $\mathrm{QED}_{3}$ as particular cases. These are given by

$$
\mathcal{L}=-\frac{1}{4} F_{\mu \nu}\left[\frac{4}{(-\square)^{\alpha}}\right] F^{\mu \nu}-e j^{\mu} A_{\mu}+\mathcal{L}_{m}
$$

where $0 \leq \alpha<1$. For a proper choice of the gauge condition, the $\mathrm{U}(1)$ vector field satisfies the equation

$$
\square^{1-\alpha} A^{\mu}=e j^{\mu},
$$


which is pseudodifferential for $\alpha \neq 0$. For $\alpha=0$, the theory above is just Maxwell $\mathrm{QED}_{3}$. In the previous section, we have shown that the case $\alpha=1 / 2$, namely PQED, is relevant for the description of the electromagnetic interactions of two-dimensional systems. In this case, Eq. (13) provides a full description of the real electromagnetic interaction for electrons confined on a plane [5].

In the above Lagrangian, the first term reads

$$
F_{\mu \nu}(\eta) \int d^{3} \eta^{\prime} \int \frac{d^{3} k}{(2 \pi)^{3}} \frac{e^{-i k \cdot\left(\eta-\eta^{\prime}\right)}}{\left(k^{2}\right)^{\alpha}} F^{\mu \nu}\left(\eta^{\prime}\right)
$$

where $k=(\mathbf{k}, \omega)$ (we excluded the index "3D" for simplicity) and $\eta=(\mathbf{r}, \tau)$. The non-locality of the propagator is a consequence of the dimensional reduction performed in order to generate the $(3+1) \mathrm{D}$ propagator within $(2+1)$ D space. A similar fact occurs when we integrate out parts of the system degrees of freedom as, for instance, in the Caldeira-Leggett model for dissipative quantum mechanics [11].

Nevertheless, in spite of being non-local, the theories described by Eq. (13) do respect causality. Indeed, it has been shown that the classic (retarded and advanced) Green functions vanish outside of the light-cone for any $\alpha$, thus preserving causality [7]. For the special case of $\alpha=1 / 2$, the classic Green functions reduce to a delta function on the light-cone surface [7]. The interesting consequence of this property is that the theory will obey Huygens principle in this case [3, 7], while $\mathrm{QED}_{3}$ does not obey it.

We see that the theories described by Eq. (13) satisfy causality despite the apparent non-locality, but it is not a priori obvious whether they respect unitarity. In the present work, we shall test the unitarity of those theories through the application of the optical theorem.

\section{UNITARITY AT TREE LEVEL}

Let us investigate here the unitarity of the theories given by Eq. (13) by considering the free Feynman propagator (tree level) in connection to the optical theorem. We use the Feynman prescription $k^{2} \rightarrow k^{2}+i \varepsilon$ in order to define the gauge field propagator corresponding to (13)

$$
G_{F}^{\mu \nu}(t, \mathbf{r})=\frac{1}{4} P^{\mu \nu} D_{F}(t, \mathbf{r})
$$

where

$$
P_{\mu \nu}=g_{\mu \nu}-\frac{\partial_{\mu} \partial_{\nu}}{\square^{2}}
$$

is the transverse projector, $g_{\mu \nu}$ is the Minkowski metric, and $D_{F}(t, \mathbf{r})$ is the corresponding scalar propagator, in the Minkowski space. Thus, we replace $\tau$ by $t$, therefore we have

$$
D_{F}(t, \mathbf{r})=\int \frac{d \omega}{2 \pi} \int \frac{d^{2} k}{(2 \pi)^{2}} \frac{e^{-i \omega t} e^{i \mathbf{k} \cdot \mathbf{r}}}{\left(\omega^{2}-\mathbf{k}^{2}+i \varepsilon\right)^{1-\alpha}}
$$

This integral has been calculated in Ref. 7] (see Appendix 1 therein), yielding

$$
D_{F}(t, \mathbf{r})=-\frac{C_{\alpha}}{\left(t^{2}-\mathbf{r}^{2}-i \epsilon\right)^{1 / 2+\alpha}},
$$

where

$$
C_{\alpha}=\frac{2^{2 \alpha-1 / 2}}{(2 \pi)^{3 / 2}} \frac{\Gamma(\alpha+1 / 2)}{\Gamma(1-\alpha)} .
$$

In order to probe the unitarity of the theories described by Eq. (13), let us first consider the scalar field. Later on we shall return to the vector field case.

Taking the amplitude corresponding to the operator Eq. (2) evaluated between states $|i\rangle$ and $|f\rangle$, which is written as $\langle i|T| f\rangle=(2 \pi)^{3} \delta^{3}\left(k_{i}-k_{f}\right) D_{i f}$ and introducing a complete set of intermediate states $|x\rangle$ on the righthand side (rhs), the above unitarity condition becomes Ref. [2]

$$
D_{i f}^{*}-D_{i f}=-i \sum_{x} \int d \Phi(2 \pi)^{3} \delta^{3}\left(k_{i}-k_{f}\right)\left(D_{i x}^{*} D_{x f}\right) \text {, }
$$

where $d \Phi$ is the phase space factor, which is needed for dimensional reasons and also to ensure that the sum over the intermediate states corresponds to the identity. The equation above is known as the generalized optical theorem.

Now, for $i \rightarrow f$, the amplitude $D_{i i}$ becomes the Feyman propagator,

$$
D_{i i}=D_{F}\left(t-t^{\prime}, \mathbf{r}-\mathbf{r}^{\prime}\right)
$$

which is given by Eq. (19). Notice that, in the Heisenberg picture $D_{F}\left(t-t^{\prime}, \mathbf{r}-\mathbf{r}^{\prime}\right)=\left\langle\mathbf{r}, t \mid \mathbf{r}^{\prime}, t^{\prime}\right\rangle$.

The unitarity condition, therefore, would lead to the equation

$$
\begin{array}{r}
D_{F}^{*}(t, \mathbf{r})-D_{F}(t, \mathbf{r})= \\
-i \int d \Phi(2 \pi)^{3} \delta^{3}(0) \int \frac{d t_{x}}{2 \pi} \int \frac{d^{2} r_{x}}{(2 \pi)^{2}} \\
D_{F}^{*}\left(t_{x}, \mathbf{r}_{x}\right) D_{F}\left(t-t_{x}, \mathbf{r}-\mathbf{r}_{x}\right) .
\end{array}
$$

Our strategy to test unitarity of a given theory will be to check whether the corresponding propagator satisfies the optical theorem. For this purpose, we Fourier transform the above equation to energy-momentum space,

$$
D_{F}^{*}(\omega, \mathbf{k})-D_{F}(\omega, \mathbf{k})=-i \mathcal{T}^{\gamma} D_{F}^{*}(\omega, \mathbf{k}) D_{F}(\omega, \mathbf{k}),
$$

where $D_{F}(\omega, \mathbf{k})$ is promptly obtained from Eq. (18), it is given by

$$
D_{F}(\omega, \mathbf{k})=\frac{1}{\left(\omega^{2}-\mathbf{k}^{2}+i \varepsilon\right)^{1-\alpha}}
$$

In the Eq. (22), we used the fact that the phase space integral combined with $\delta^{3}(0)$ yields $\mathcal{T}^{\gamma}$, where $\mathcal{T}$ is the 
characteristic time of the system and $\gamma=-2(1-\alpha)$ (see App. A).

Defining $\chi_{\alpha}=\left(\omega^{2}-\mathbf{k}^{2}+i \varepsilon\right)^{1-\alpha}$, we can write the equation above as

$$
\frac{2 \operatorname{Im}\left(\chi_{\alpha}\right)}{\chi_{\alpha}^{*} \chi_{\alpha}}=\frac{\mathcal{T}^{-2(1-\alpha)}}{\chi_{\alpha}^{*} \chi_{\alpha}}
$$

For unitarity to be respected, we must have

$$
2 \operatorname{Im}\left(\chi_{\alpha}\right)=\mathcal{T}^{-2(1-\alpha)}
$$

However, since the rhs is a constant, for the above condition to be consistent, $\operatorname{Im}\left(\chi_{\alpha}\right)$ must also be a constant, in the limit $\varepsilon \rightarrow 0$. In other words, in that limit the lefthand side (lhs) can not be a function of $\lambda=\omega^{2}-\mathbf{k}^{2}$ for Eq. (25) to be consistent.

In order to verify this condition, we introduce a polar representation for $\chi_{\alpha}$, namely, $\chi_{\alpha}=\left(\rho e^{i \theta}\right)^{1-\alpha}$, with $\rho^{2}(\lambda)=\lambda^{2}+\varepsilon^{2}$ and $\theta(\lambda)=\sin ^{-1}(\varepsilon / \rho)$. Then, we require that

$$
\frac{d}{d \lambda} \operatorname{Im}\left(\chi_{\alpha}\right)=\frac{d}{d \lambda} \rho \sin [(1-\alpha) \theta]=0
$$

Calculating the derivative, we obtain

$$
\tan [\theta(\lambda)(1-\alpha)]=\tan [\theta(\lambda)]
$$

which has an obvious solution $\alpha=0$. Indeed, it is clear that for this value of $\alpha, \operatorname{Im}\left(\chi_{0}\right)=\varepsilon$ and therefore is independent of $\lambda$.

A less obvious solution is $\alpha=1 / 2$, which is valid because in this case Eq. (27) admits a solution $\theta(\lambda)=2 \pi-\varepsilon$, which is compatible with the definition of $\theta(\lambda)$. In this case, we also find $\operatorname{Im}\left(\chi_{1 / 2}\right)=\varepsilon$ (see App. B).

We conclude that, for the theories whith $\alpha=0$ and $\alpha=$ $1 / 2$, the two sides of Eq. (24) would coincide consistently by identifying $2 \varepsilon$ with $\mathcal{T}^{-2}$. For other values of $\alpha, \operatorname{Im}\left(\chi_{\alpha}\right)$ would depend on $\lambda$ and, therefore, we would not be able to find a consistent solution of Eq. (25) satisfying the generalized optical theorem.

The demonstrations provided above were meant for the scalar theories associated with Eq. (13). The corresponding results for the vector propagator Eq. (16) then, follow straightforwardly by making $\mathcal{T}^{-2(1-\alpha)} / 4 \rightarrow \mathcal{T}^{\prime-2(1-\alpha)}$ and from the fact that the transverse projector has the property: $P^{2}=P$.

We conclude that out of the class of theories described by Eq. (13), only the ones with $\alpha=0$ and $\alpha=1 / 2$, namely $\mathrm{QED}_{3}$ and PQED are unitary.

\section{UNITARITY OF PQED IN THE RPA APPROXIMATION}

Next, we consider PQED, the case for which $\alpha=1 / 2$. As we have seen, it describes the EM interaction of the particles coupled to it. Having graphene in mind we describe the electrons as massless Dirac fermions experiencing the EM interaction mediated by the gauge field $A_{\mu}$. The Lagrangian in this case reads [12]

$$
\mathcal{L}=\frac{1}{4} F_{\mu \nu}\left[\frac{4}{\sqrt{-\square}}\right] F^{\mu \nu}+\bar{\psi}\left(i \not \partial+e \gamma^{\mu} A_{\mu}\right) \psi,
$$

where $e$ is the dimensionless coupling constant, $\psi$ is the Dirac field, and $\gamma^{\mu}$ are Dirac matrices which can be either two or four dimensional, since we are in $(2+1) \mathrm{D}$.

The corrections to the gauge-field propagator are expressed in terms of the the vacuum polarization $\Pi_{\mu \nu}(p)$. The one-loop expression for this has been calculated in Ref. [13] and is given by

$$
\Pi_{\mu \nu}(k)=-\frac{e^{2} \sqrt{k^{2}}}{16} P_{\mu \nu}(k)+\frac{e^{2}}{2 \pi}\left(n+\frac{1}{2}\right) \epsilon_{\mu \nu \alpha} k^{\alpha},
$$

where $n$ is an integer. The result above is for two dimensional Dirac matrices.

According to Eq. (18), the free gauge field propagator in momentum space reads

$$
G_{0, \mu \nu}(k)=\frac{1}{4} \frac{P_{\mu \nu}(k)}{\sqrt{k^{2}}} .
$$

We include the vacuum polarization corrections by using the random phase approximation (RPA), where the corrected propagator is given by the geometrical series

$$
\begin{aligned}
G_{\mu \nu}= & G_{0, \mu \alpha}\left[\delta_{\alpha, \nu}+\Pi^{\alpha \beta} G_{0, \beta \nu}+\right. \\
& \left.\Pi^{\alpha \beta} G_{0, \beta \sigma} \Pi^{\sigma \gamma} G_{0, \gamma \nu}+\ldots\right] .
\end{aligned}
$$

Because of the peculiar momentum dependence of the vacuum polarization tensor, the corrected propagator has basically the same momentum dependence as the free one

$$
G_{\mu \nu}(k)=\frac{1}{\sqrt{k^{2}+i \epsilon}}\left(A_{1} P_{\mu \nu}(k)+A_{2} \frac{\epsilon_{\mu \nu \alpha} k^{\alpha}}{\sqrt{k^{2}}}\right),
$$

where $A_{1}$ and $A_{2}$ are constants depending on the coefficients of the vacuum polarization tensor. Note that we use the Feynman prescription as we did before. Unitarity of the theory is guaranteed provided that the optical theorem Eq. (20) is still respected.

The propagator above can be conveniently written as

$$
G_{\mu \nu}(k)=C_{\mu \nu}(k) D_{F}(k),
$$

where

$$
C_{\mu \nu}(k)=A_{1} P_{\mu \nu}(k)+A_{2} \frac{\epsilon_{\mu \nu \alpha} k^{\alpha}}{\sqrt{k^{2}}},
$$

with $D_{F}(k)$ given by the Eq. (18) for $\alpha=1 / 2$.

The optical theorem now reads

$$
\begin{array}{r}
G_{\mu \nu}^{*}(t, \mathbf{r})-G_{\mu \nu}(t, \mathbf{r})= \\
-i \int d \Phi(2 \pi)^{3} \delta^{3}(0) \int \frac{d t_{x}}{2 \pi} \int \frac{d^{2} r_{x}}{(2 \pi)^{2}} \\
G_{\mu \alpha}^{*}\left(t_{x}, \mathbf{r}_{x}\right) G_{\alpha \nu}\left(t-t_{x}, \mathbf{r}-\mathbf{r}_{x}\right) .
\end{array}
$$


Next, we adopt the same strategy as for the noninteracting case and perform a Fourier transform in both sides of the above equation, again, considering that the Fourier transform of a convolution is a product. We obtain

$$
G_{\mu \nu}^{*}(\omega, \mathbf{k})-G_{\mu \nu}(\omega, \mathbf{k})=-i \mathcal{T}^{-1} G_{\mu \alpha}^{*}(\omega, \mathbf{k}) G_{\alpha \nu}(\omega, \mathbf{k}) .
$$

The lhs of Eq. (36) is given by

$$
\frac{C_{\mu \nu}(k) 2 i \operatorname{Im}\left(\chi_{1 / 2}\right)}{\left[\left(\omega^{2}-\mathbf{k}^{2}\right)^{2}+\epsilon^{2}\right]^{1 / 2}}
$$

whereas the rhs of Eq. (36) reads

$$
\frac{-i \mathcal{T}^{-1} C_{\mu \alpha}(k) C_{\alpha \nu}(k)}{\left[\left(\omega^{2}-\mathbf{k}^{2}\right)^{2}+\epsilon^{2}\right]^{1 / 2}}
$$

where

$$
C_{\mu \nu}^{2}(k)=\left(A_{1}^{2}-A_{2}^{2}\right) P_{\mu \nu}(k)-2 A_{1} A_{2} \frac{\epsilon_{\mu \nu \alpha} k^{\alpha}}{\sqrt{k^{2}}} .
$$

We now consider Eq. (37) and Eq. (38). Since both are proportional to the operators $P_{\mu \nu}(k)$ and $\epsilon_{\mu \nu \alpha} k^{\alpha} / \sqrt{k^{2}}$, therefore, we have to compare the corresponding coefficients of both terms. Using the result of App. B, we conclude that the optical theorem will be obeyed and consequently, unitarity preserved, provided we make the choices

$$
(2 \varepsilon)^{1 / 2}=\frac{A_{1}^{2}-A_{2}^{2}}{2 A_{1}} \mathcal{T}^{-1},
$$

in the $P_{\mu \nu}(k)$ term and

$$
\left(2 \varepsilon^{\prime}\right)^{1 / 2}=A_{1} \mathcal{T}^{-1},
$$

in the $\epsilon_{\mu \nu \alpha} k^{\alpha} / \sqrt{k^{2}}$ term.

This concludes our proof of the unitarity of PQED of massless electrons in the RPA approximation.

\section{BEYOND THE RPA APPROXIMATION}

Within the RPA approximation, the one-loop expression for the vacuum polarization tensor, Eq. (29) is used in the geometrical series that corrects the free propagator of the gauge field. This approach can be improved by adding the two-loop correction for the vacuum polarization tensor, as calculated by Teber [6],

$$
\Pi_{\mu \nu}^{(2)}(k)=-\frac{\sqrt{k^{2}}}{16}\left(\frac{92-9 \pi^{2}}{18 \pi}\right) \alpha_{g} P_{\mu \nu},
$$

where $\alpha_{g} \approx 300 / 137=2.189$ is the fine structure constant of graphene. Considering that $\left(92-9 \pi^{2}\right) / 18 \pi \approx$ 0.056 , we see that the two-loop correction is sensible. There is no correction to the Chern-Simon term due to the Coleman-Hill theorem [14].
Observe that, remarkably, the two-loop correction has precisely the same functional dependence as the one-loop one. As a consequence, the only effect of the two-loop correction to the vacuum polarization is to redefine the constant $A_{1}$ in Eq. (32). Therefore, it immediately follows that the optical theorem, and consequently, unitarity are respected in the two-loop extension of the RPA approximation.

\section{CONCLUSIONS}

We have tested the unitarity of a class of field theories in $2+1 \mathrm{D}$ containing fractional powers $(1-\alpha)$ of the d'Alembertian operator, which despite being nonlocal, respect causality. $\mathrm{QED}_{3}$ and $\mathrm{PQED}$ are particular cases, respectively, with $\alpha=0$ and $\alpha=1 / 2$.

Our strategy is to verify whether the propagator satisfies the optical theorem. We first considered the free propagator for generic $\alpha$ and showed that only for $\alpha=0$ and $\alpha=1 / 2$, namely, for $\mathrm{QED}_{3}$ and PQED, unitarity is respected. Inspection of the propagators in Eqs. (19) and (23) shows that one theory is dual to the other. Indeed, for $\alpha=0$ the exponent in $D_{F}(\omega, \mathbf{k})$ is unity, whereas the one in $D_{F}(t, \mathbf{r})$ is $1 / 2$. For $\alpha=1 / 2$, the same occurs, but with $\mathbf{k} \rightarrow \mathbf{r}$ and $\omega \rightarrow t$.

We then considered the case of PQED coupled to massless Dirac fermions, which is the model for graphene. We have shown that the propagator corrected both within the RPA approximation and in its two-loop extension satisfy the optical theorem, hence unitarity is preserved in both cases.

\section{ACKNOWLEDGMENTS}

This work was supported in part by CNPq (Brazil), CAPES (Brazil), FAPERJ (Brazil), The Netherlands Organization for Scientific Research (NWO) and by the Brazilian government project Science Without Borders. We are grateful to G.'t Hooft for interesting discussions.

\section{APPENDIX A: THE PHASE SPACE FACTOR}

Here we are going to determine the phase space factor 1]. Let us consider Eq. (21) and write

$$
\int d \Phi(2 \pi)^{3} \delta^{3}(0) \equiv \mathcal{T}^{\gamma}
$$

where $\mathcal{T}$ is the characteristic time scale of the system. For dimensional reasons, we have $\gamma+3=2(\alpha+1 / 2)$ and consequently $\gamma=-2(1-\alpha)$. This justifies the $\gamma-$ dependence in Eq. (22). 


\section{APPENDIX B: THE $\operatorname{Im}\left(\chi_{\alpha}\right)$}

Let us show here that, for $\alpha=0,1 / 2$, indeed, the expression of $\operatorname{Im}\left(\chi_{\alpha}\right)$ relevant for the optical theorem, is given by $\varepsilon, \varepsilon^{1 / 2}$, respectively, and therefore just depends on $\varepsilon$.

Using $\chi_{\alpha} \equiv\left(\omega^{2}-\mathbf{k}^{2}+i \varepsilon\right)^{1-\alpha}$, we have, for $\alpha=0$, $\chi_{0}=\left(\omega^{2}-\mathbf{k}^{2}+i \varepsilon\right)$ and evidently $\operatorname{Im}\left(\chi_{0}\right)=\varepsilon \propto \mathcal{T}^{-2}$.

For the case $\alpha=1 / 2$, notice that the condition for the optical theorem to be satisfied is

$$
\frac{2 \operatorname{Im}\left(\chi_{1 / 2}\right)}{\left[\left(\omega^{2}-\mathbf{k}^{2}\right)^{2}+\varepsilon^{2}\right]^{1 / 2}}=\frac{K \mathcal{T}^{-1}}{\left[\left(\omega^{2}-\mathbf{k}^{2}\right)^{2}+\varepsilon^{2}\right]^{1 / 2}}
$$

for some dimensionless constant K. Squaring this equation and multiplying both the numerators by $\varepsilon$, we obtain both sides proportional to $\delta\left(\omega^{2}-\mathbf{k}^{2}\right)$. As a consequence, we must equate the numerators at $\omega^{2}-\mathbf{k}^{2}=0$, namely,

$$
\left.2 \operatorname{Im}\left(\chi_{1 / 2}\right)\right|_{\omega^{2}=\mathbf{k}^{2}}=(2 \varepsilon)^{1 / 2}=K \mathcal{T}^{-1}
$$

which completes the proof for $\alpha=1 / 2$.
[1] M. E. Peskin and D. V. Schroeder, An Introduction to Quantum Field Theory, Westview (1995). M. Kaku, Quantum Field Theory A Modern Introduction, Oxford University Press (1993). Steven Weinberg, The Quantum Theory of Fields Vol. I Foundations, Cambridge University Press (1995).

[2] Matthew Schwartz, Introduction to Quantum Field Theory, Havard University (2008).

[3] C.G.Bollini and J.J. Giambiagi, J. Math. Phys. 34, 610 (1993).

[4] A. H. Castro Neto, F. Guinea, N. M. R. Peres, K. S. Novoselov and A. K. Geim, Rev. Mod. Phys. 81, 109 (2009).

[5] E. C. Marino, Nucl. Phys. B 408, 551 (1993).

[6] S. Teber, Phys. Rev. D 86, 025005 (2012).

[7] R. L. P. G. do Amaral and E. C. Marino, J. Phys. A:
Math and Gen 25, 5183 (1992).

[8] A. Kovner and B. Rosenstein, Phys. Rev. B 42, 4748 (1990); N. Dorey and N. E. Mavromatos, Nucl. Phys. B 386, 614 (1992).

[9] E. C. Marino, Phys. Lett. B 263, 63 (1991).

[10] Van Sérgio Alves, Walace S. Elias, Leandro O. Nascimento, Vladimir Juričić and Francisco Peña, Phys. Rev. D 87, 125002 (2013).

[11] A. Caldeira and A. Leggett, Ann. of Phys. 149, 374 (1983).

[12] E. C. Marino, Leandro O. Nascimento, Van Sérgio Alves, C. Morais Smith, arXiv:1309.5879.

[13] A. Coste and M. Luscher, Nucl. Phys. B 323, 631 (1989).

[14] M. D. Bernstein and T. Lee, Phys. Rev. D 32, 4 (1985); S. Coleman and B. Hill, Phys. Lett. B 159, 184 (1985). 